\title{
CCAAT Enhancer-binding Protein Alpha (CEBPA) Gene Expression in a Cohort of Turkish Patients with Multiple Myeloma
}

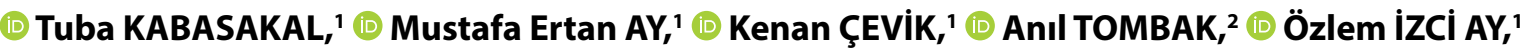 \\ (1) Mehmet Emin ERDAL' ${ }^{1}$
}

'Department of Medical Biology and Genetics, Mersin University, Mersin-Turkey

${ }^{2}$ Department of Hematology and Oncology, Mersin University, Mersin-Turkey

\begin{abstract}
OBJECTIVE
Hematopoiesis is regulated and maintained by the function of the particular transcription factors. CCAAT enhancer-binding protein alpha (CEBPA), an important transcription factor, has a part in the regulation of cell cycle, granulocytic differentiation, and more. The possible role of CEBPA in multiple myeloma (MM) development has not been clarified so far. Therefore, this study aimed to explore the relationship between the expression levels of the CEBPA gene in Turkish patients diagnosed with MM.

\section{METHODS}

Using quantitative real-time PCR, the expression level of the CEBPA gene was examined in the bone marrow samples of $44 \mathrm{MM}$ patients and 13 healthy controls. Statistical analyses were performed using SPSS, 13.3.1, and $\mathrm{p}<0.05$ was evaluated as statistical significance level.

RESULTS

Although there was a decrease CEBPA expression levels in the patient group compared to the control group, no statistically significant relationship was found in terms of CEBPA gene expression level and MM compared with the controls $(\mathrm{p}=0.436)$.

\section{CONCLUSION}

Our findings suggest that the change in the expression level of CEBPA transcript itself has probably no effect in the pathogenesis of MM patients, although it is an important problem that needs further researches with large-scale samples.
\end{abstract}

Keywords: Bone marrow; CEBPA; gene expression; multiple myeloma; transcription factor.

Copyright $\odot$ 2022, Turkish Society for Radiation Oncology

\section{Introduction}

Multiple myeloma (MM), also known as plasma cell dyscrasia, is the second most common hematological malignancy that accounts for approximately $10-15 \%$ of all hematological cancers. It is characterized by clonal expansion of plasma cells/plasmablasts originating from abnormal precursor lymphoid B-cell lineage leading to overproduction of non-functional monoclonal immunoglobulin ( $\mathrm{M}$ proteins) and impaired hematopoiesis.[1,2] The main clinical symptoms of MM include infection, bone destruction, anemia, immunosuppression, and kidney failure.[3] The incidence rate of this malignancy has been dramatically increased in recent years and is five/100,000 in Europe. It frequently affects elderly people, and the average age at 
diagnosis is around 65-66 years. Conventionally, MM is associated with a poor prognosis, with the median overall survival ranges from a few months to more than 10 years. [4,5] Up to now, the basic mechanism causing plasma cell disorders and progression to MM has not been fully executed. Many factors promoting tumor formation may play a role in the formation multistage process of MM, which is a biologically highly heterogeneous disease accompanied by many genomic changes. The genetic alterations occurring in the development of MM start at the a premalignant phase, termed monoclonal gammopathy of undetermined significance (MGUS), where M proteins are synthesized, continuing throughout the stages of intermediate disease stage between MGUS and MM, termed smoldering MM (SMM), MM, and plasma cell leukemia (clonal evolution of plasma cell dyscrasias). [4]

The malignant transformation of B-cell or plasma cell into MGUS and subsequently MM requires both an initiating event and multiple secondary genetic events. Initiating events are broadly subdivided into IgH translocations or hyperdiploidy. In the continuum of disease stages, genetic lesions accumulate in the tumor clone. Although there are still unknown points in the formation and progression of $M M$, upregulation of cyclin D expression appears to be a necessary occurrence early stage of MM. The next stage in MM etiology is non-random trisomies, CNVs, translocations containing IgH, FGFR3, MMSET, transcription factors (MAF and MAFB), inactivation of tumor suppressor genes ( $\mathrm{pRB}$ and $\mathrm{p} 53$ ), and activation of oncogenic genes (MYC and RAS), deletions, and other chromosomal rearrangements.[6]

Transcription factors, due to their wide range of cellular functions, can be shown among the genes that are expected to be affected first in this process.[7] Like all immune cells, B-cells are derived from hematopoietic stem cells that primarily develop in the bone marrow. Hematopoietic stem cells can successively differentiate into multipotent progenitors, common lymphoid progenitors, and eventually mature $B$ cells through the stages pre-pro- $B$, pro- $B$, pre- $B$, immature $B$, and transitional $\mathrm{B}$-cells. This process requires the many transcription factors including E2A, PU.1, and PAX5 as well as interleukin 7 cytokine signaling.[6] CCAAT enhancerbinding protein alpha (CEBPA) gene is located on chromosome 19q13.1 and CEBPA protein that is produced by this gene is a member of the transcription factors family including a basic leucine zipper motif.[8,9] It is mostly expressed in hepatocytes, adipocytes, and myeloid cells of the hematopoietic system. In the hematopoietic sys- tem, CEBPA is important for stem and progenitor cell function and regulates the differentiation of hematopoietic cells and is necessary for the generation of the granulocyte monocyte progenitor within the bone marrow and thereby, formation of mature eosinophils, neutrophils, and monocytes.[10,11] It is also essential for many vital biological processes involving in the regulation of genes expressed in the cell cycle, apoptosis, differentiation of hematopoietic stem cells, and more; within these processes, it could act not only as a transcription activator but also repressor, depending on cell type and target genes.[12,13] In addition to its important functions in myelopoiesis, it also contributes to early B lymphoid development. In the B-cells that do not encounter antigens and are transcriptionally quiescent, genes encoding transcription factors begin to be transcribed with antigenic stimulation. CEBPA; it may be one of the transcription factors activated in this process and it can be thought that changes in CEBPA expression may play a role in the etiology of MM. Given that CEBPA has been identified to play crucial roles in the regulation of normal hematopoietic system, it is not surprising that the changes of CEBPA expression level lead to the development of MGUS, SMM, and MM transition.

Thus, our aim was to evaluate whether the expression levels of CEBPA display in bone marrow samples from patients diagnosed with $\mathrm{MM}$ and the bone marrow of healthy controls using qPCR method.

\section{Materials and Methods}

This study was approved by Mersin University Health Sciences Ethics Committee with the decision numbered $2018 / 469$, dated $21 / 11 / 2018$. The study was planned and conducted in accordance with the Helsinki Declaration principles.

\section{Patients}

For this investigation, bone morrows of 44 patients diagnosed with MM (17 females and 27 males) were collected from the department of hematology-oncology and 13 healthy control bone marrows (eight males and five females). Bone marrow samples of the control group had been taken from the sternum of individuals who have not taken any hematological disease diagnosis during cardiac surgery (aspirated from surgical area and not used in any diagnosis and treatment) recruited from the Department of Cardiac Surgery, Mersin University Hospital, Mersin, Turkey. The suitability of the number of individuals in the patient and control groups in- 
cluded in our study was statistically tested with "power analysis" before the study in terms of the effectiveness of the study (data not provided). Written informed consent was obtained from all participants before their participation. Our study was approved by the Medical Sciences Ethical Committee of Mersin University. The study was planned and conducted in accordance with the principles of the Declaration of Helsinki.

\section{Total RNA Isolation and cDNA Synthesis}

First, the total RNA was isolated from bone marrow of patients and healthy individuals using TRIzol reagent (Invitrogen). Following isolation, the quality of all RNA samples was checked using a NanoDrop spectrophotometer (Thermo Scientific, Wilmington, DE, USA). All samples demonstrated high purity (OD 260/280 $\mathrm{nm}$ ratio $>1.8$ ). Second, the reverse transcription reaction was performed to obtain cDNA using an automated Thermal Cycler (Veriti, Applied Biosystems). The reaction conditions were as follows: $37^{\circ} \mathrm{C}$ for 60 min and $95^{\circ} \mathrm{C}$ for $5 \mathrm{~min}$. A total cDNA reaction volume of $50 \mu \mathrm{L}$ included $2 \mu \mathrm{g} / \mu \mathrm{L}$ of RNA sample, $200 \mathrm{U} /$ $\mu \mathrm{L}$ RevertAid Reverse Transcriptase $(0.2 \mu \mathrm{L}$ ) (Thermo Scientific, Vilnius, Lithuania), $5 \times$ RT buffer $(8 \mu \mathrm{L}), 5$ $\mu$ poly-T primer $(5 \mu \mathrm{L}), 2 \mathrm{mM}$ each of dNTPs $(20 \mu \mathrm{L})$, $40 \mathrm{U} / \mu \mathrm{l}$ of RiboLock RNase inhibitor $(0.5 \mu \mathrm{L}$ ) (Thermo Scientific, Vilnius, Lithuania), and nuclease-free water.

\section{Real-Time PCR Analysis}

Quantitative analysis of CEBPA gene was run on an ABI Prism 7500 Real-Time PCR System (Applied Biosystems) using the following primers and probes used in the present study; F: 5' CAAATATTTTGCTTTATCAGCCGATA-3' and R: 5'-CGCACATTC ACATTGCACAA-3' Prob: 5'-FAMACACTTGTATCTGGCCTCTGTGCCCCA-BHQ1-3' for CEBPA; F: 5'-GGCACCCAGCACAATGAAG-3' and R: 5-GCCGATCCACACGGAGTACT-3', Prob: 5-Yakima Yellow TCAAGATCATTGCTCCTCCTGA GCGC-BHQ-1-3' for the housekeeping gene, beta-actin (ACTB). All reactions were conducted in 20 $\mu \mathrm{L}$ final volume including $12.5 \mu \mathrm{L}$ of TaqMan Gene Expression Master Mix (Applied Biosystems), $5 \mu \mathrm{L}$ cDNA, $2.5 \mu \mathrm{L} 900 \mathrm{nmol}$ each primer, $1 \mu \mathrm{L} 200 \mathrm{nmol}$ of each TaqMan ${ }^{\circ}$ probes, and nuclease-free water. PCR parameters were started after pre-incubation at $50^{\circ} \mathrm{C}$ for $2 \mathrm{~min}$ and denaturation at $95^{\circ} \mathrm{C}$ for $10 \mathrm{~min}$ followed by 50 cycles of $95^{\circ} \mathrm{C}$ for $15 \mathrm{~s}$ and $60^{\circ} \mathrm{C}$ for $1 \mathrm{~min}$. Trials were conducted in duplicate for each data point. The $2^{-\Delta \Delta C t}$ method was used to measure changes in the gene expression detected by qPCR analysis.

\section{Statistical Analysis}

Statistical analyses were performed using the Statistica 13.3.1 software. Shapiro-Wilk test was used to test the normality assumption and to analyze the homogeneity of variance, Levene's test was performed. The nonparametric method, Mann-Whitney U-test, was used to compare two groups for non-normal distributions. Numerical data were summarized as mean (standard deviation) or median (percentiles). The relationship between categorical variables was evaluated using Chisquare statistics. Statistical significance level was determined as $\mathrm{p}<0.05$.

\section{Results}

The expression level of the CEBPA gene was successfully evaluated in 44 patients diagnosed with $\mathrm{MM}$ and 13 healthy volunteers. The patient group consisted of 17 women and 27 men (47-91-years-old), with a mean age of $65.89 \pm 10.02$ and the control group consisted of five women and eight men whose ages ranged from 22 to 80 (mean age of $62.00 \pm 14.60$ ).

The mRNA expression level of the selected gene according to age and gender of the studied cohort was rated and no statistically significant correlation was observed depending on these two parameters ( $\mathrm{p}=0.276$ and $\mathrm{p}=0.870$, respectively).

The analysis also revealed that the median CEBPA expression level in MM patients was downregulated from the level found in the control group (Fig. 1). However, the statistical evaluation of our results demonstrated that there was not a significant difference regarding selected gene expression between the two groups $(\mathrm{p}=0.436)$.

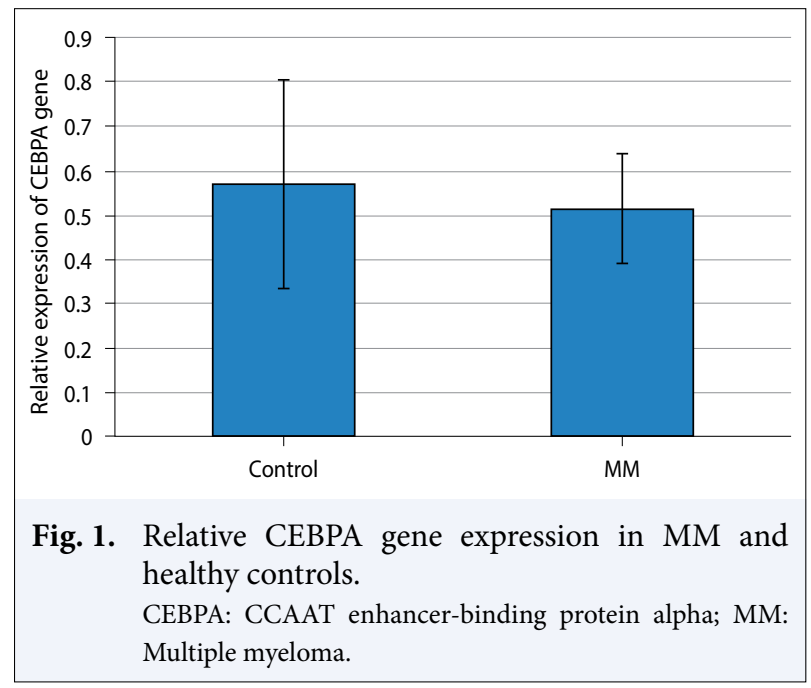




\section{Discussion}

Although several chromosomal rearrangements, epigenetic changes, nucleotide variations, and transcriptional alterations have provided significant insight into MM pathogenesis, our knowledge about molecular mechanism of this malignancy still remains incomplete. We still need "new genes" for early diagnosis and preventive therapy for MM which also may provide a reference for identification of the novel therapeutic pathways. Here, the present study was performed to answer the question whether the expression level of CEBPA gene affects the process of development/progression in patients diagnosed with MM. CEBPA is a multifunctional transcription factor. Dysregulation of CEBPA expression has been identified in various human neoplasms, including lung, liver, and breast cancer but is best characterized in hematological malignancies.[14,15] Targeted inhibition of CEBPA gene in mice has exhibited its contribution to cellular differentiation. Knockout mice models have defined a crucial role for CEBPA in hematopoietic tissue. For example, a research conducted by Hasemann et al.[16] demonstrated that loss of CEBPA leads to aberrant self-renewal of hematopoietic stem cells and cell death. $\mathrm{C} / \mathrm{EBPa}$ is expressed mainly in myeloid cells and its default in CEBPA-/- mice results in the complete absence of granulocytic differentiation with an arrest at the stage of immature myeloblasts.[17] Besides its central role in myeloid malignancies, a previous study by Chapiro et al.[18] also reported its role in B-cell malignancies. On the other hand, Collombet et al.[19] reported that CEBPA is required at the onset of lymphoid specification. The first relationship between CEBPA gene and MM was reported by Fuchs et al.[20] who found genomic mutations to generate in the CEBPA gene in $5.1 \%(2 / 39)$ of $M M$ cases. This finding suggests that dysregulation of this gene may have a role in the pathogenesis of MM by blocking the ability of B-cells to properly differentiate. In the present cross-sectional study, the obtained results demonstrated that the expression level of the CEBPA gene was similar to the level found between MM and healthy subjects. Conflicting results have been published from previous reports concerning the CEBPA expression in MM patients. Zang et al.[21] investigated the expression level of CEBPA gene in bone marrow cells of 69 elderly patients with $M M$ and they found significantly lower CEBPA expression and protein level compared with 38 healthy volunteers. In consistent with our results, they also reported that CEBPA gene expression was not correlated with sex and age. In contrast to the previous study, Han et al.[22] demonstrated increased CEBPA gene expression in peripheral blood monocytes of MM patients. It should be stated that MM is defined as a genetically and clinically highly complex and heterogeneous disease, represented by the presence of the distinct numbers of recurrent genetic defects, a subclonal evolution pattern, and different clinical characteristics. Hence, the plausible causes of these disagreements could be the smaller sample size, the high heterogeneity of this malignancy, the lack of MM ISS stage, and the other clinical parameters except age and sex.

\section{Conclusion}

To the best of our knowledge, this is the first investigation in the literature to look at CEBPA gene expression level and how it relates to MM development/progression in a cohort of Turkish patients with MM. The obtained results suggested that CEBPA gene expression level may not be effective involve in MM development. To compare our results, further studies in this field are needed on larger groups of MM patients from different populations.

Acknowledgments: The authors thank the patients and the control persons for their participation in this study. This work was supported by the Mersin University Research Foundation under Grant BAP-SBE 2019-1-TP2-3206.

Peer-review: Externally peer-reviewed.

Conflict of Interest: All authors declared no conflict of interest.

Ethics Committee Approval: The study was approved by the Mersin University Health Sciences Ethics Committee (No: 2018/469, Date: 21/11/2018). The study was planned and conducted in accordance with the Helsinki Declaration principles.

Financial Support: This study has received no financial support.

Authorship contributions: Concept - M.E.A., T.K.; Design - M.E.A., T.K., A.T.; Supervision - M.E.E., Ö.İ.A., M.E.A.; Funding - None; Materials - A.T.; Data collection and/or processing - T.K., K.Ç.; Data analysis and/or interpretation - M.E.A., T.K., A.T., Ö.İ.A., K.Ç.; Literature search - T.K.; Writing - M.E.A., T.K., K.Ç.; Critical review - Ö.İ.A.

\section{References}

1. Smith CJ, Ambs S, Landgren O. Biological determinants of health disparities in multiple myeloma. Blood Cancer J 2018;8(9):1-7. 
2. Weinhold N, Heuck CJ, Rosenthal A, Thanendrarajan $\mathrm{S}$, Stein CK, Van Rhee F, et al. Clinical value of molecular subtyping multiple myeloma using gene expression profiling. Leukemia 2016;30(2):423-30.

3. Prideaux SM, O'Brien EC, Chevassut, TJ. The genetic architecture of multiple myeloma. Adv Hematol 2014;2014:864058.

4. Soliman AM, Lin TS, Mahakkanukrauh P, Das S. Role of microRNAs in diagnosis, prognosis and management of multiple myeloma. Int J Mol Sci 2020;21(20):7539.

5. Manier S, Salem KZ, Park J, Landau DA, Getz G, Ghobrial IM. Genomic complexity of multiple myeloma and its clinical implications. Nat Rev Clin Oncol 2017;14(2):100-13.

6. Barwick BG, Gupta VA, Vertino PM, Boise LH. Cell of origin and genetic alterations in the pathogenesis of multiple myeloma. Front Immunol 2019;10:1121.

7. Neuse CJ, Lomas OC, Schliemann C, Shen YJ, Manier $\mathrm{S}$, Bustoros $\mathrm{M}$, et al. Genome instability in multiple myeloma. Leukemia 2020;34(11):2887-97.

8. Pabst T, Mueller BU. Complexity of CEBPA dysregulation in human acute myeloid leukemia. Clin Cancer Res 2009;15(17):5303-7.

9. Szmajda D, Krygier A, Jamroziak K, ŻebrowskaNawrocka M, Balcerczak E. Expression level of CEBPA gene in acute lymphoblastic leukemia individuals. Sci Rep 2019;9(1):15640.

10. Barberi T, Cui C, Friedman, AD. C/EBPa induces Ebf1 gene expression in common lymphoid progenitors. PLoS One 2020;15(12):e0244161.

11. Johnson PF. Molecular stop signs: Regulation of cellcycle arrest by C/EBP transcription factors. J Cell Sci 2005;118(12):2545-55.

12. Gholami M, Bayat S, Manoochehrabadi S. Investigation of CEBPA and CEBPA-AS genes expression in acute myeloid leukemia. Rep Biochem Mol Biol 2019;7(2):136.

13. Barbagallo D, Condorelli AG, Piro S. CEBPA exerts a specific and biologically important proapoptotic role in pancreatic $\beta$ cells through its downstream network targets. Mol Biol Cell 2014;25(16):2333-41.

14. Koschmieder S, Halmos B, Levantini E, Tenen DG. Dysregulation of the $\mathrm{C} / \mathrm{EBP} \alpha$ differentiation pathway in human cancer. J Clin Oncol 2009;27(4):619-28.

15. Lourenço AR, Coffer PJ. A tumor suppressor role for $\mathrm{C} / \mathrm{EBPa}$ in solid tumors: More than fat and blood. Oncogene 2017;36(37):5221-30.

16. Hasemann MS, Lauridsen FK, Waage J, Jakobsen JS, Frank AK, Schuster $\mathrm{MB}$, et al. $\mathrm{C} / \mathrm{EBPa}$ is required for long-term self-renewal and lineage priming of hematopoietic stem cells and for the maintenance of epigenetic configurations in multipotent progenitors. PLoS Genet 2014;10(1):e1004079.

17. Jones LC, Lin ML, Chen SS, Krug U, Hofmann WK, Lee $\mathrm{S}$, et al. Expression of C/EBP $\beta$ from the C/ebp a gene locus is sufficient for normal hematopoiesis in vivo. Blood 2020;99(6):2032-6.

18. Chapiro E, Russell L, Radford-Weiss I, Bastard C, Lessard M, Struski S, et al. Overexpression of CEBPA resulting from the translocation $t(14 ; 19)$ (q32;q13) of human precursor $\mathrm{B}$ acute lymphoblastic leukemia. Blood 2006;108(10):3560-3.

19. Collombet S, van Oevelen C, Ortega JL, Abou-Jaoudé W, Di Stefano B, Thomas-Chollier M, et al. Logical modeling of lymphoid and myeloid cell specification and transdifferentiation. Proc Natl Acad Sci USA 2017;114(23):5792-9.

20. Fuchs O, Provaznikova D, Kocova M, Kostecka A, Cvekova P, Neuwirtova R, et al. CEBPA polymorphisms and mutations in patients with acute myeloid leukemia, myelodysplastic syndrome, multiple myeloma and non-Hodgkin's lymphoma. Blood Cells Mol Dis 2008;40(3):401-5.

21.Zang YZ, Chen XL, Bai YL, Zhang WH, Yu RH. Expression and clinical significance of $\mathrm{C} / \mathrm{EBP} \alpha$ gene in elderly multiple myeloma. Zhongguo Shi Yan Xue Ye Xue Za Zhi 2019;27(1):123-8.

22. Han X, Zhou DB, Duan MH, Hua BL, Wang X, Zhang $\mathrm{JP}$, et al. $\mathrm{C} / \mathrm{EBPa}$ in multiple myeloma patients may lead to increased hepcidin. Zhongguo Shi Yan Xue Ye Xue Za Zhi 2014;22(5):1316-20. 